\title{
\%
}

\section{METODOLOGÍA DE LA INVESTIGACIÓN} TECNOLÓGICA EN INGENIERÍA

\author{
Methodology of techcological research in engineering
}

Celso De La Cruz Casaño1

${ }^{1}$ Universidad Continental, Unidad de Investigación de la Facultad de Ingeniería

Correo electrónico: cdelacruz@continental.edu.pe

\begin{abstract}
Resumen-En las universidades peruanas es bien conocida la metodología de la investigación científica; sin embargo, es poco conocida la metodología de la investigación tecnológica.

La investigación tecnológica, conocida también como desarrollo tecnológico, se encarga de la creación de nuevos procesos, sistemas o máquinas; o la mejora de éstos.

En el presente trabajo se muestra la metodología de la investigación tecnológica, las diferencias con la metodología de la investigación científica, su importancia para el desarrollo de las industrias y ejemplos prácticos relacionados a las carreras de ingeniería.
\end{abstract}

Palabras clave: Investigación tecnológica, desarrollo tecnológico, inventar, innovar, ingeniería

\section{INTRODUCCION}

La investigación, según Sampieri [1], "es un conjunto de procesos sistemáticos, críticos y empíricos que se aplican al estudio de un fenómeno". Sin embargo, esta definición no abarca a la investigación tecnológica, la cual está más preocupada por transformar la realidad que por darle una explicación o comprenderla [2]. Es decir, la investigación tecnológica no se limita a estudiar un fenómeno natural que existe en la naturaleza, sino a crear nuevas realidades que no existen, para ello utiliza el proceso de invención, innovación, diseño o de desarrollo tecnológico [3, 4].

La importancia de la investigación tecnológica radica en que los desarrollos tecnológicos pueden convertirse en innovaciones, que se aplican directamente a las empresas haciéndolas más productivas y competitivas, necesarias para el desarrollo de un país. A mayor productividad en las empresas. mayor recaudación tendrá el Estado y podrá invertir más en investigación, cerrándose un círculo virtuoso.

Para obtener innovaciones, en la mayoría de veces, se requiere realizar investigación científica cuyos conocimientos generados darán paso a las investigaciones tecnológicas, éstas a su vez culminan en innovaciones; por lo tanto se debe invertir tanto en la investigación científica como en la tecnológica. Las empresas privadas que patentan innovaciones
Abstract- At Peruvian universities the scientific methodology of research is well known; however, the technologic methodology of research is almost unknown.

The technologic methodology, known also as technologic development, permits the creation of new process, systems or machines; or the improvement of these.

In the present work the methodology of technologic research, the differences with the methodology of scientific research, its relevance for the development of industries and practice engineering examples are presented.

Keywords: Technological research, technological development, invention, innovation, engineering.

también pueden obtener beneficios a través de las regalías Así, estas empresas pueden continuar invirtiendo en investigación, cerrándose también un círculo virtuoso.

En la mayoría de universidades peruanas se reconoce sólo la metodología de la investigación científica, sin embargo, una parte de los alumnos y docentes tienen interés en realizar investigación tecnológica, sobre todo en las carreras de ingeniería. Al desconocer el método de investigación tecnológica, ellos terminan utilizando el método de investigación científica y se desvían de sus verdaderos objetivos.

En el presente trabajo se presenta la metodología de la investigación tecnológica, la cual servirá de guía para orientar a los investigadores que deseen realizar investigaciones de este tipo. Los ejemplos que se brindan son de investigaciones internacionales que evidencian la realización de investigaciones tecnológicas a nivel mundial.

El artículo se organiza de la siguiente manera: primero se exponen las definiciones de la investigación tecnológica, luego se muestran las diferencias entre la investigación científica y la tecnológica citando ejemplos de investigaciones tecnológicas publicadas a nivel internacional, finalmente se presenta el proceso de la investigación tecnológica basado en autores y se propone un proceso de investigación tecnológica para las carreras de ingeniería. 


\section{INVESTIGACIÓN TECNOLÓGICA}

Según Cegarra [3], la investigación tecnológica, también denominada "desarrollo", tiene por finalidad la invención de artefactos o de procesos con el objeto de ofrecerlos al mercado y obtener un beneficio económico.

Salvo excepciones, el desarrollo suele efectuarse en la empresa, requiriendo en algunos casos el concurso de la universidad, centros estatales o privados para llevar a buen término algunos aspectos de su investigación.

Según García [2], la principal diferencia del paradigma tecnológico respecto a los paradigmas clásicos (cuantitativo y cualitavio) es que en esta visión del mundo estamos más preocupados por transformar la realidad que por darle una explicación o comprenderla.

Sin embargo, esto no significa que no se esté interesado en conocer la realidad, sino que el conocimiento que se busca obtener, extraído de la realidad, sea operativo o ejecutable, esto es, debe orientar la ejecución de acciones concretas que nos beneficien como seres humanos, tanto en lo ingenieril-empresarial como en lo social.

El fin de la labor tecnológica es apoyarse en el conocimiento para lograr la transformación de una realidad concreta particular. En el paradigma tecnológico tenemos un proceso que integra la investigación y la transformación a la vez, es decir requerimos conocer el objeto de estudio para después intervenir en una realidad particular modificando el estado de cosas, hasta alcanzar una aproximación a lo deseado.

Según Rincón [5], mientras que la ciencia hace referencia exclusiva a la generación de conocimientos nuevos, a través de la investigación, la técnica y la tecnología buscan la aplicación de los conocimientos derivados de investigaciones científicas; es decir, la técnica, la ciencia y la tecnología se diferencian por los objetivos que persiguen, sobre todo, en la forma de hacer las cosas para la satisfacción de las necesidades humanas, la producción de bienes y servicios (tecnología), mientras que, la ciencia pretende responder y entender la naturaleza y la sociedad.

\section{DIFERENCIAS ENTRE INVESTIGACIÓN} CIENTÍFICA Y TECNOLÓGICA

En el libro de García [4] se muestran las diferencias entre el método cuantitativo, cualitativo y tecnológico. A continuación, se presentan las principales diferencias.

\begin{tabular}{|c|c|c|}
\hline Cuantitativo & Cualitativo & Tecnológico \\
\hline $\begin{array}{c}\text { La realidad existe, } \\
\text { es externa al suje- } \\
\text { to y es constante. }\end{array}$ & $\begin{array}{c}\text { La realidad se cons- } \\
\text { truye, interactúa } \\
\text { con el sujeto y es } \\
\text { cambiante. }\end{array}$ & $\begin{array}{c}\text { La realidad se puede } \\
\text { cambiar y el hombre } \\
\text { puede llevar a cabo } \\
\text { tal intervención. }\end{array}$ \\
\hline $\begin{array}{c}\text { Cómo es el mun- } \\
\text { do. }\end{array}$ & $\begin{array}{c}\text { Cómo viven o qué } \\
\text { significa para ellos ... }\end{array}$ & $\begin{array}{c}\text { Cómo transformar } \\
\text { esa parte del mundo. }\end{array}$ \\
\hline $\begin{array}{c}\text { Genera afirmacio- } \\
\text { nes universales } \\
\text { teórico-explica- } \\
\text { tivas. }\end{array}$ & $\begin{array}{c}\text { Consigue afirma- } \\
\text { ciones particulares } \\
\text { descriptivo- inter- } \\
\text { pretativas-com- } \\
\text { prensivas. }\end{array}$ & $\begin{array}{c}\text { Determina afirma- } \\
\text { ciones particulares } \\
\text { operativas-ejecuta- } \\
\text { bles. }\end{array}$ \\
\hline
\end{tabular}

En el cuadro se puede observar que la investigación tecnológica modifica la realidad, es decir el fin es crear nuevas máquinas, estructuras, softwares, procesos, etc., así como también desarrollar mejoras sobre las existentes.

Como ejemplo de estas investigaciones se puede mencionar al trabajo de Sengupta [6], el cual desarrolla un nuevo algoritmo para explorar el espacio de alternativas de diseño de forma automática. Este algoritmo se puede utilizar en la optimización del diseño de un sistema electrónico, eléctrico, mecánico, etc. Otro ejemplo es el trabajo de Cho [7], en el cual se diseña un sistema de ventilación con una unidad de limpieza y control de demanda para una edificación multirresidencia.

Este diseño está basado en la demanda para ahorrar energía en la ventilación, reduce el suministro de aire exterior utilizando un filtro de aire mientras se mantiene la calidad del aire; en esta operación el sistema de ventilación mide la concentración de $\mathrm{CO} 2$ y $\mathrm{HCHO}$.

El trabajo previo [8] también se puede tomar como ejemplo de investigación tecnológica. En esta investigación se desarrolló un sistema de navegación para una silla de ruedas robótica basada en landmarks.

El sistema propuesto utiliza los sensores en las ruedas, segmentos metálicos como landmarks y un identificador por radio frecuencia (RFID) para ubicar al robot en el edificio, y así poder realizar la navegación automática calculando el camino más corto para llegar a su destino.

El sistema de navegación también incluye una estrategia para evadir obstáculos: desvía al robot de su trayectoria y, una vez evadido el obstáculo, éste vuelve a su trayectoria original.

\section{PROCESO DE INVESTIGACIÓN TECNOLÓGICA}

En el libro de García [4], se proponen las siguientes etapas para realizar una investigación tecnológica:

1. Observar. En esta etapa intervienen los conocimientos previos, la experiencia adquirida y la intuición del observador.

2. Determinar el problema. Se determina qué es lo que ocurre o está presente y requiere de atención.

3. Documentar. Es reunir la información pertinente; esto permite conocer, identificar y relacionar datos para tomar decisiones basadas en el conocimiento.

4. Reflexionar. En esta etapa se generan algunas respuestas al problema o se formula uno nuevo con base en la información obtenida en la etapa previa.

5. Elaboración del proyecto de intervención. En una investigación tecnológica no se diseña un proyecto de investigación, como ocurre en la investigación científica, sino que se formula un plan para efectuar una intervención en la realidad.

6. Valorar. Se evalúa la efectividad de un proyecto de intervención mediante la presentación y discusión de la propuesta o la experimentación de la propuesta.

7. Comunicar. Se comunica cuál será la participación de los involucrados en la implementación de la propuesta. 
8. Implementar. Se ejecuta el plan de trabajo para implementar la propuesta.

9. Seguimiento. Se asegura que se cumpla el plan de trabajo.

10. Evaluación. Se evalúa si los objetivos se lograron convenientemente.

Otra propuesta se puede encontrar en Rincón [5], la cual se resume en el siguiente índice de contenidos:

1. Resumen

2. Preámbulo

3. Introducción

4. Contextualización y problemática. Situación inicial -dimensión deseada-, situación final. Objetivos. Justificación.

5. Teoría histórica, técnica y operativa. Sustentaciones teóricas histórico-evolutivas, científicas, técnicas-tecnológicas, empíricas y bases legales.

6. Metodología investigativa. Descripción del proceso de invención, innovación u optimización. Explicación del diseño (invención, diseño, innovación). Procesos para la creación del diseño. Recursos: instrumentos, herramientas, materiales, inversiones (análisis de costos).

7. Análisis y resultados. Explicación de objetivos: Experimentos, ensayos, pruebas, evaluaciones, seguimientos, estadísticas.

8. Prototipo. Estudio de rentabilidad, factibilidad y viabilidad. Evaluación del prototipo. Proyecciones y prospectivas de la innovación (mejoras, optimización, transformación)

9. Conclusiones

10. Aportes y recomendaciones

A continuación se propone una metodología de investigación tecnológica adaptada a las carreras de Ingeniería, basadas en las metodologías presentadas anteriormente.

\section{Planteamiento del estudio}

a. Planteamiento del problema.

El problema a diferencia de la investigación científica, parte de una necesidad.

b.Formulación del problema.

La formulación del problema, a diferencia del método de investigación científica, puede ser en forma de lista de requerimientos y restricciones (como en [6]) o en forma de pregunta.

c. Objetivos

d. Justificación e importancia

\section{Marco teórico}

a. Antecedentes del problema

b. Bases teóricas.

c. Definición de términos básicos

\section{Hipótesis.}

Se plantea una o varias soluciones tentativas al problema de investigación.

\section{Metodología}

a. Plan de trabajo del diseño o desarrollo. En esta etapa se explican los pasos a seguir para alcanzar los objetivos.

b. Recursos. Instrumentos, herramientas, materiales e inversiones.

\section{Implementación}

En esta etapa se ejecuta el diseño o desarrollo.

\section{Evaluación}

En esta etapa se evalúa el diseño o desarrollo. La prueba puede ser mediante simulaciones o mediante prototipos.

La hipótesis podría no ser cierta, puesto que en el proceso de investigación el diseño o desarrollo podría tomar múltiples formas, es muy complicado estimar con certeza los resultados al inicio de la investigación.

Se puede observar en el método anterior que no se consideran las variables del objeto de estudio al inicio de la investigación como se hace en la investigación científica. La razón es que la investigación científica estudia un fenómeno de una realidad existente y, por lo tanto, requiere de las variables al inicio del estudio.

En la investigación tecnológica se crea una nueva realidad, por eso las variables cambiarán de acuerdo a cómo evoluciona el diseño o desarrollo.

A continuación se muestra un ejemplo de la formulación del problema, objetivo, hipótesis y plan de trabajo del diseño o desarrollo en el método de investigación tecnológica.

\section{Formulación del problema}

Se requiere un sistema de ventilación que mantenga el aire fresco y ahorre energía para un conjunto habitacional.

\section{Objetivo}

Diseñar un sistema de ventilación que mantenga el aire fresco y ahorre energía para un conjunto habitacional.

\section{Hipótesis}

- Un sistema de control mantendrá de manera adecuada el aire fresco.

- Un sistema de control que minimiza la ventilación requerida y a la vez garantiza la calidad del aire disminuye el consumo de energía.

El sistema de control adecuado utiliza un sensor de CO2. 
- La recirculación de aire con un filtro para evitar utilizar aire exterior permite ahorrar energía.

\section{Plan de trabajo del diseño o desarrollo:}

- Se diseñará el sistema de control que cumpla con los requerimientos y restricciones.

- Se seleccionará el sensor de CO2 adecuado y se buscarán otras alternativas para medir la calidad de aire en el sistema de control.

- Se diseñará un sistema de recirculación automática del aire utilizando un filtro y se buscarán otras alternativas para disminuir la energía gastada en la ventilación.

Como se puede observar en el ejemplo anterior, utilizar el método propuesto facilita enormemente la elaboración de un proyecto de investigación del tipo tecnológico, dado que los puntos tratados en el ejemplo se dan de manera natural en la solución de problemas en ingeniería.

\section{CONCLUSIONES}

El desarrollo tecnológico se viene dando con gran fuerza en investigaciones a nivel mundial.

La investigación tecnológica a diferencia de la científica, modifica la realidad y no solo la estudia.

La investigación tecnológica tiene gran importancia porque los desarrollos tecnológicos mejoran la productividad de las industrias.

El método de investigación propuesto facilita enormemente la elaboración de un proyecto de investigación tecnológica.

\section{AGRADECIMIENTOS}

El autor agradece a la Universidad Continental por brindarle los recursos necesarios para realizar la presente investigación.

\section{REFERENCIAS BIBLIOGRÁFICAS}

[1] R. Hernández Sampieri, C. Fernández y M. Baptista, Metodología de la Investigación. 5a ed, México: McGraw Hill, 2010.

[2] F. García Córdova y R. Muñoz, "El conocimiento como recurso sustantivo del cambio tecnológico en las organizaciones", Criterio Libre, vol. 7, n. ${ }^{\circ} 11,2009$.

[3] J. Cegarra Sánchez, Metodología de la investigación científica y tecnológica. Madrid: Díaz de Santos, 2004.

[4] F. García Córdova y M. R. Trejo, La perspectiva de la investigación tecnológica en educación. México: Limusa, 2012

[5] I. B. Rincón, "Investigación científica y tecnológica como factores de innovación", Entelequia: Revista Interdisciplinar, $\mathrm{n}^{\circ} 14,2012$

[6] A. Sengupta y S. Bhadauria, "Automated design space exploration of multi-cycle transient fault detectable datapath based on multi-objective user constranints for application specific computing" Advances in Engineering Software, vol. 82, 2015.

[7] W. Cho, D. Song, S. Hwang y S. Yun, "Energy-efficient ventilation with air-cleaning mode and demand control in a multi-residential building," Energy and Building, vol. 90, 2015

[8] C. De La Cruz, W. Cardoso y T. Freire, "A robust navigation system for robotic wheelchairs", Control Engineering Practice, vol.19, 2011 